\title{
L'écoute musicale et ses enjeux dans Conversations avec le maître de Cécile Wajsbrot
}

\section{Annelies Schulte Nordholt ${ }^{1}$}

Accepted: 26 September 2020 / Published online: 24 October 2020

(c) The Author(s) 2020

\begin{abstract}
Conversations avec le maître, the first volume of a series called 'Haute mer', is a novel about the experience of listening to music. Through conversations between a composer and an anonymous young woman, it conveys crucial aspects of musical listening and of its verbal expression. This article examines the functioning of dialogue between the two characters; moreover, it studies the relationship between creation and reception, as expressed in the novel. It also examines what is at stake in this dialogue: the power of art in its relation to reality, to History. How may music become the echo of contemporary catastrophes? The article offers a close reading of the novel's descriptions of listening to music: what literary techniques enable the author to put it into words?
\end{abstract}

Keywords Wajsbrot, Cécile · Conversations avec le maître · Musical listening · Musical ekphrasis

\section{Introduction}

Conversations avec le maître (2007) est le premier volume d'un cycle romanesque intitulé Haute mer. Dans la postface du roman, Cécile Wajsbrot en explique la thématique générale:

Dans cette série de romans, je voudrais explorer la question de la création, prenant en compte, certes, le point de vue du créateur, mais aussi le point de vue des autres, ceux que nous sommes tous - auditeurs de musique, visiteurs de galeries ou d'expositions, et lecteurs. (2007, 173).

C'est dire l'ambition de ce cycle de cinq romans, dont chaque volume met en scène un ou plusieurs arts différents. Il n'explore pas seulement les trois arts traditionnels - la musique, la littérature et la peinture-mais l'art dans toute sa diversité

Annelies Schulte Nordholt

a.e.schulte@hum.leidenuniv.nl

1 Université de Leiden, Leiden, The Netherlands 
contemporaine: musique classique dans Conversations avec le maitre, sculpture dans L'Ile aux musées, photographie et chanson dans Totale éclipse, art vidéo dans Sentinelles. ${ }^{1}$ De plus, dans le volume final, Destruction, plusieurs arts-ou peut-être faut-il dire médias - sont mis en dialogue, dans une vision dystopique d'une société qui a banni l'art. Il y a dans cette entreprise une ambition de totalisation qui semble assez à contre-courant de notre époque. Elle rappelle les grandes esthétiques de l'Idéalisme allemand, notamment celle de Hegel et nous ramène en amont de Hegel, aux Romantiques et à leur conception de l'art comme un absolu. Je reviendrai plus loin à ce lien de l'œuvre de Wajsbrot avec l'esthétique du Romantisme allemand.

Cependant, l'exploration de Wajsbrot ne s'effectue pas par l'essai philosophique mais par le roman qui, loin de tout exposé systématique, est vécu comme une aventure: «une fois commencé, le récit nous mène dans des zones incertaines qu'il serait absurde de vouloir déterminer à l'avance.» (173). Ecrire, c'est s'aventurer «en haute mer», comme un «navigateur du large» (Beaune-la-Rolande, 2004, 52), vers des terres inconnues, loin du port. En outre, par son caractère narratif, le roman permet une dramatisation de l'expérience du récepteur. Et c'est cette orientation sur la réception qui fait la profonde originalité du cycle. Car s'il existe une multitude de romans mettant au centre un grand artiste_Le chef d'œuvre inconnu de Balzac, L'œuvre de Zola, Le Docteur Faustus de Thomas Mann, La carte et le territoire de Michel Houellebecq, pour ne citer que les plus connus-il en existe peu dont le protagoniste se trouve de l'autre côté, celui de la réception. Et il en est moins encore dont la protagoniste est féminine.

On peut se demander d'où vient l'intérêt de Wajsbrot pour le versant de la réception. Il y a certes une raison quantitative: si les créateurs, les artistes sont peu nombreux, le public, par contre, est sans nombre: il s'agit de nous tous. N'est-ce pas déjà une raison pour ne pas négliger leur expérience ? Une autre raison, plus prégnante, est qu'en tout artiste, il y a un créateur et un récepteur. On peut imaginer que Wajsbrot ait voulu mettre en scène le dialogue entre ces deux instances, dialogue intérieur à tout artiste. Cependant, l'auteur a choisi le genre du roman, avec des personnages en chair et en os, et non celui du dialogue philosophique, ce qui rend cette hypothèse moins convaincante.

Une autre réponse encore est que le récepteur-plus que le créateur-est un être plongé de plain-pied dans le monde, dans l'Histoire. Contrairement au créateur qui peut (qui devrait peut-être) garder ses distances par rapport au monde, le récepteur est acculé au réel. Lorsqu'il/elle regarde une œuvre plastique, écoute une chanson ou une symphonie, lit un livre, il est contraint de se poser en permanence une question: comment cette œuvre adhère-t-elle au monde, à notre époque? Dans quelle mesure est-elle, comme on dit en allemand, «zeitgemäss»? C'est une question que Wajsbrot a développée dans son essai «Le son du silence» (2008a), où elle se positionne par rapport à la littérature française d'après-guerre. Elle y marque ses distances par rapport aux avant-gardes artistiques des années 1960-70, du Nouveau Roman à Oulipo. Elle leur reproche en bloc d'avoir tourné le dos au monde réel et de s'être réfugié

\footnotetext{
1 Dans l'ordre, le cycle comprend les volumes suivants: Conversations avec le maître (2007), L'île aux musées (2008), Sentinelles (2013), Totale éclipse (2014), Destruction (2019).
} 
dans le formalisme et le repli sur soi (2008a, 245). Face à ces avant-gardes, partisanes d'un art qui ne prend plus en charge le monde, elle y prône un roman qui s'ouvre pleinement sur celui-ci et sur l'Histoire, et qui forme par là même une jonction entre le contenu et la forme littéraire.

Or si on examine l'œuvre de Wajsbrot dans son ensemble, il y a un mot qui, pour elle, résume l'expérience-clef qui marque notre époque et investit la vie quotidienne de ses personnages: c'est la notion de «catastrophe», ou de désastre. C'est un terme qui pourra étonner par son caractère apocalyptique et son pathos, mais il parcourt en filigrane ses romans, depuis les tout premiers. En effet, dans Beaune-la-Rolande (2004), Mémorial (2005) et La Trahison (2005), Wajsbrot, qui descend de Juifs polonais émigrés en France dans les années 1920, et dont le grand-père a été déporté, interroge la mémoire de la Shoah et de Vichy, tout particulièrement dans la perspective des générations nées après. Cependant, refusant de se voir éternellement comme «gardienne du temple» (Wajsbrot 2008b, 28), Wajsbrot souligne volontiers que de l'eau a passé sous les ponts depuis 1945, et que l'écrivain doit témoigner non seulement du passé, mais aussi de sa propre époque, donc du $\mathrm{XXI}^{\mathrm{e}}$ siècle:

Nous sommes dans l'après-coup. Et le désastre est devant nous, la catastrophe est annoncée, elle porte d'autres noms, des noms qui ne sont plus des noms de lieu, des noms de villes comme au XXe siècle [allusion, bien entendu, à Auschwitz] mais des noms communs, des expressions: changement climatique, effet de serre, émeutes de la faim - tsunami. (Wajsbrot 2008a, 248).

C'est cette catastrophe-imminente et même déjà en cours, à en croire son roman Destruction — qui, en dernière instance, fait l'urgence et l'actualité de l'interrogation de Wajsbrot sur la réception de la musique et des autres arts. Cette interrogation devient centrale dans le dernier volume, Destruction, mais elle est présente dès Conversations avec le maitre. Il s'agira donc, dans ce qui suit, d'étudier ce récit de l'écoute musicale en en développant la thématique, les enjeux et les procédés. Dans un premier temps, on analysera le dialogue entre créateur et auditrice: que nous apprend-il sur leur interaction, à la fois à l'intérieur et au-delà de l'intrigue? Sur leur implication dans l'Histoire? Et plus généralement, comment l'art-et la musique en particulier-peut-il être en consonance avec le réel et l'Histoire ? Dans un deuxième temps, on s'attachera plus particulièrement aux descriptions de l'audition musicale: comment ce récit parvient-il à raconter, à décrire l'expérience de l'écoute, s'ingéniant à exprimer les sons par un autre medium, les mots? Ce dernier point m'amènera à lire certains passages du roman comme une ekphrasis musicale, et à en examiner le fonctionnement.

\section{La narratrice et le 'maître'}

Conversations avec le maître est le récit des conversations quotidiennes d'une jeune femme anonyme avec un compositeur également sans nom, qui est de vingt ans son aîné. Récit à la première personne, fait par l'auditrice. Pendant une longue période, elle a fait des visites rituelles au compositeur: chaque jour, après son travail, elle 
monte le voir pendant une heure, et prend des notes en rentrant. Jusqu' au jour où elle apprend par téléphone qu'il a mis fin à ses jours. Un ou deux ans après, un disciple du maître lui demande de «rassembler les souvenirs qu'elle a de lui» (2007, 33), ce qui résulte en le récit que nous avons en main, qui est désigné comme «un témoignage, une reconstitution» (29).

Ce compositeur, elle l'appelle en pensée «le maître»: il maestro, titre traditionnellement donné à un grand directeur d'orchestre ou à un compositeur. Ce terme suggère un rapport d'inégalité entre les deux personnages: le grand compositeur, l'artiste mûr-notons bien, de genre masculin — versus la jeune femme, qui travaille dans une agence immobilière, métier aux antipodes de l'art. Cependant, ce rapport est moins hiérarchique qu'il ne semble. Certes, cette conversation est en réalité un monologue du maitre, où la jeune femme ne fait qu'écouter et poser une question de temps en temps. Pourtant, son statut n'est pas celui d'une admiratrice ou groupie:

Il était le maître mais je n'étais pas une disciple, je n'étais pas un de ces jeunes artistes qui cherchent un aîné prestigieux pour apprendre leur art et s'introduire dans un milieu - j'étais sans visage, anonyme, un membre de l'auditoire. (27).

De plusieurs manières, la jeune femme est l'auditrice: elle écoute la musique dont lui parle le maître, mais aussi ses propos. Elle est ainsi doublement l'auditrice. C'est sa faiblesse, mais aussi sa force. Ces conversations n'ont d'ailleurs pas lieu à sa demande, mais à celle du maître, qui l'a invitée un jour, par besoin d'avoir un interlocuteur, de partager son expérience des affres de la création artistique. En plus, comme on a vu, c'est elle qui mène le récit, puisqu' elle en est la narratrice, ce qui lui donne aussi une fonction critique, outre celle de témoin, de dépositaire de la mémoire du maître.

Malgré le caractère hiérarchique de leur relation, les deux interlocuteurs sont donc dans une relation d'interdépendance. Si elle se sent initialement attirée par le compositeur, par son aura d'artiste, lui en revanche a besoin d'elle comme d'une caisse de résonance pour ses réflexions et un remède à sa solitude. Cependant, son amour tacite pour le maître se termine par une déception lorsqu' elle s'aperçoit que le maître reste éternellement à distance. Auprès de cet être cérébral, ce qui lui manque cruellement, ce sont les sentiments, c'est une complicité; elle attend de lui «une explication-pourquoi m'avait-il choisie — une reconnaissance, le fait qu'entre nous, quelque chose sans nom ni forme existait.» (59) Or cette expérience physique, sensuelle, seule la musique_qu'ils partageront—pourra la lui donner, la musique dans son lien intime au plaisir mais aussi à la souffrance. Le maître est conscient de ce lien, mais il ne parvient pas à le vivre, d'où peut-être sa stérilité et son échec final.

La narratrice, elle, n'est pas seulement à l'écoute du maitre, mais aussi des autres. Son travail quotidien la plonge dans le monde d'aujourd'hui, avec ses crises. Tout en travaillant pour les privilégiés, et se sachant elle-même une privilégiée, pourvue d'un toit et d'un emploi, elle est extrêmement perméable, sensible à la souffrance des «réprouvés», proches et lointains. L'histoire se passe en 2004, lors du tsunami dans le Sud-Est asiatique. Par son écran d'ordinateur, tous les soirs, la jeune femme absorbe les images du désastre et lit les avis de recherche des survivants sur les réseaux sociaux. Le roman analyse avec lucidité cette manière assez nouvelle (surtout en 2007, quand le roman a paru) de percevoir le 
monde par le biais des écrans, et comment cela a affecté et même profondément modifié notre rapport au monde extérieur. Pour la narratrice, l'écran est certes un précieux réceptacle qui la relie au monde mais il est aussi «ce qui nous en sépare» (39); il permet de voir les visages des naufragés, de connaître leurs noms, d'entendre les voix des survivants mais ces voix sont «déconnectées» (52), car immatérielles, sans corps. Sur internet, nous «prenons part au désastre» (68) mais c'est une souffrance qui a un côté voyeuriste car ce ne sont pas «nos morts». Une souffrance qui reste impuissante précisément parce qu'elle passe par l'image: «Je vois les oubliés, les perdus, les visages se fondent et se déforment-nous voyons trop de choses, envahis, submergés d'images que nous ne demandons pas.» (95).

Cependant, dans le roman, les «réprouvés» de la société ne sont pas seulement un spectacle lointain, ils sont aussi tout proches, ils nous entourent, tentant à tout moment de violer notre égoïsme quotidien. C'est le sens du personnage de la jeune fille «venue des confins de l'Ukraine», qui hante les alentours de l'agence où travaille la narratrice; elle est en quête de secours et peut-être d'une habitation d'un prix accessible. Contrairement aux victimes du tsunami et aussi au maître, elle a une présence matérielle, corporelle: «Cette jeune femme vient me dire que le corps existe car dans sa présence absolue, sa silhouette muette, seul le corps parle et répare les années d'oubli.» (52) Avec elle, c'est le sort des milliers d'immigrés illégaux à Paris qui s'impose à la conscience: leur ségrégation, leur isolement, leur misère quotidienne. Par sa présence insistante, elle lance un appel à la narratrice, qui n'a rien d'une sainte: c'est une femme normale, qui vit sa vie et qui hésite, comme la plupart des gens, entre des élans de générosité et une réaction de repli. Ici, le roman analyse avec subtilité les contradictions de la générosité et de l'altruisme. Un jour, la narratrice invite la jeune fille au café pour l'écouter tranquillement. C'est le même café où elle a jadis rencontré le maitre. Or en entrant, «je me dirigeai avec résignation vers la place du maître, qui était libre, m'installant de son côté, alors que la jeune femme s'asseyait à mon ancienne place.» (83). Cet élan de générosité renforce donc paradoxalement l'inégalité entre les deux femmes, mettant la narratrice dans une périlleuse position de supériorité. C'est elle qui est maintenant devenue «le maître».

Par un art achevé de la composition, le roman tisse des liens entre tous ces «réprouvés»: la jeune fille d'Ukraine est vue comme «une rescapée d'un tsunami intérieur» (68) et même le maître, rejeté par ses collègues compositeurs, est un réprouvé, un naufragé que la narratrice compare aux victimes du tsunami (72). Et après coup, la narratrice voit sa propre histoire avec le maître comme une sorte de «tsunami» personnel. Cette mise en parallèle des personnages et de leur histoire s'effectue par l'enchevêtrement des différents fils du récit: sans solution de continuité, les lambeaux de conversation avec le maître alternent avec les apparitions répétées de la jeune fille d'Ukraine et les réflexions de la narratrice. Ainsi, à un moment donné, le maître lui parle d'un quatuor qu'il aurait écrit et dont la structure est assez complexe: «quatre mouvements en alternance selon le système des rimes embrassées» (73). Or cette structure est à l'image de celle du récit de Wajsbrot, où les différents fils du récit sont savamment enchevêtrés: les conversations avec le maître sur la musique, les rencontres avec la jeune fille, à l'agence immobilière et les réflexions de la narratrice. La continuité entre ces diverses composantes est encore renforcée 
par le fait que les dialogues sont intégrés au récit, sans guillemets ni tirets: c'est une conversation rapportée après coup, intégrée aux réflexions de la protagoniste.

Par rapport au monde et à sa souffrance, le maître et la narratrice occupent donc des positions inverses et symétriques: celles du retranchement et de l'implication. Or ces positions sont déterminantes pour leur attitudes respectives vis-à-vis de la musique, que j' examinerai à présent.

\section{Pour un art enraciné dans l'Histoire}

Le maître, on l'a vu, vit en solitaire, retranché du monde. Son appartement, situé au $7^{\mathrm{e}}$ étage, et l'immense vue sur Paris depuis la baie vitrée, sont emblématiques de cette attitude retirée qui contemple le monde comme un panorama, vu de loin, à travers une vitre. Il vit «dans un autre monde, là-haut», un monde spirituel entièrement séparé du «monde d'en bas» où vit la narratrice (25). Selon ses dires, la création nécessite cet isolement total, elle exige le recueillement, afin de «s'éloigner de ce qu'on est» et de «renoncer à soi» (27). Pour lui, la musique est une quête de l'absolu, qui nécessite d'aller jusqu' au bout (46)—attitude extrême qui annonce déjà son échec final. Pour lui, l'artiste est le proverbial Prométhée voleur de feu: «je dissèque le feu, disait-il [...], je travaille sans lunettes et sans gants» (22). Dans cette zone à haut risque qu'est l'art, le maître se situe dans la lignée des artistes maudits: des artistes «qui avaient abandonné en route, qui s'étaient tus d'une façon ou d'une autre et dont on n'avait découvert l'importance qu'après leur mort.» (19) Ce sont là des clichés romantiques; ils rendent le portrait du maître un peu caricatural-ce qui ne le rend guère sympathique. Ce portrait sera d'ailleurs mis en question par la narratrice. Elle perce à jour l'égocentrisme du maître et entrevoit l'impasse où son attitude l'a mené.

Pourtant, même si le maître s'est réfugié dans cette proverbiale tour d'ivoire, a-t-il pour autant perdu tout contact avec le monde et la souffrance humaine? C'est peut-être le cas dans sa vie privée, mais nullement au niveau de la musique. En effet, son isolement volontaire est paradoxalement la condition même d'une ouverture sur le monde:

Composer, me dit un jour le maître, c'est être sur une île, non seulement parce que le travail nécessite l'isolement mais parce qu'il faut savoir s'isoler du monde et de la vie pour mieux les retrouver dans ce qu'on imagine [...] (91, je souligne).

Son ambition est de faire une œuvre parfaitement en phase avec son époque: voilà le sens du requiem qu'il projette. C'est dire que le maître considère la musiquesa musique, même si celle-ci reste virtuelle_comme un art «zeitgemäss», prenant la mesure de l'époque. Dans une de leurs conversations, il esquisse rapidement les grandes phases de l'évolution de la musique et des arts en général. Si, dans un premier temps, la peinture, la littérature et la musique visaient à «représenter le monde ou le décrire» (ce sont les Réalismes du $\mathrm{XIX}^{\mathrm{e}}$ siècle), le $\mathrm{XX}^{\mathrm{e}}$ siècle marque un «deuxième temps», où l'art va aspirer à «se représenter lui-même» (40). Maintenant, «la peinture parle de peinture, la littérature de littérature, et la musique, de 
musique.» (40-41). Il fait allusion bien entendu à l'art moderne, aux avant-gardes avec leur tendance à l'autoréflexivité et leur culte de la forme. Or le maître prend nettement position par rapport aux avant-gardes, auxquelles appartiennent ses contemporains: il leur fait de lourds reproches, notamment d'avoir perdu le contact avec le monde et avec sa souffrance. Aussi est-il à la recherche d'un «troisième temps», d'une troisième voie, qui irait au-delà des avant-gardes. Voici comment il définit cette troisième voie:

Le troisième temps pourrait être un temps de synthèse qui donnerait non une description mais une analyse, une conception du monde alliant le discours sur le monde et le discours sur l'art, une totalité. (41, je souligne).

Dans cette définition, notons premièrement, le mot «monde» répété deux fois: si les avant-gardes ont tourné le dos au monde, il s'agit maintenant de rétablir le contact avec lui. Deuxièmement, les notions de synthèse et de totalité: l'art, la musique que le maître a en tête devra être à la fois contenu («discours sur le monde») et forme («discours sur l'art»), sans négliger aucun des deux. Or ici, le maître semble se faire le porte-parole de l'auteur, de Cécile Wajsbrot qui, dans «Le son du silence», cité plus haut, tient des propos comparables à propos de la littérature. Elle y soutient que le roman d'aujourd'hui perpétue une dissociation du moi et du monde héritée des avant-gardes du XX $\mathrm{X}^{\mathrm{e}}$ siècle. Elle discerne alors deux écueils de la littérature contemporaine: ou bien le roman se fait autofiction-un genre qu'elle dénonce assez sommairement comme narcissique et subjectiviste_ ou bien il se tourne vers le monde et la société (pensons à la vogue actuelle des récits de voyage, des récits sur le quotidien) mais alors il refuse souvent tout travail sur la forme, et par la même «fait sortir le roman du champ littéraire» (Wajsbrot 2008a, 250). A cette impasse de la littérature contemporaine, elle oppose une conception autrement ambitieuse du roman: «Le roman est totalité- totalité de la forme, totalité du contenu, totalité de la forme et du contenu.» (ibid.). Or cette conception de l'œuvre d'art comme unité, synthèse de la forme et de l'idée semble lui venir du Romantisme allemand. Elle l'illustre d'ailleurs dans son essai en citant des vers de Hugo Hofmannsthal. ${ }^{2}$ Voyons maintenant comment cette conception de la musique comme synthèse fond-forme est mise en œuvre dans le roman, du côté du maître, mais aussi de la narratrice.

Le maître se distancie donc des avant-gardes musicales du $\mathrm{XX}^{\mathrm{e}}$ siècle. Cela explique le choix des œuvres qu'il fait connaître à la jeune femme: les symphonies de Chostakovitch et une œuvre du compositeur français Charles Koechlin, le mâ̂tre un peu oublié de Poulenc_-surtout auteur, lui aussi, d'œuvres symphoniques. Certes, il s'agit de compositions éminemment modernes, du $\mathrm{XX}^{\mathrm{e}}$ siècle, mais elles sont mélodiques, écrites selon les règles de l'harmonie, et non atonales par exemple. En outre, ce sont des compositions (cela vaut en tout cas pour celles de Chostakovitch) profondément enracinées dans l'Histoire, liées à ses catastrophes. Dans ce sens, elles correspondent parfaitement à ce qui est en jeu dans la musique, aux yeux du maître:

\footnotetext{
${ }^{2}$ En traduction: «Si vous séparez la forme du contenu, vous n'êtes pas des créateurs. La forme est le sens du contenu, le contenu, l'essence de la forme.» (Wajsbrot 2008a, 250).
} 
Voyez-vous, disait le maître, la musique porte plus que d'autres arts l'expression de la catastrophe. Vous qui pensez qu'elle ne parle de rien, regardez le nombre d'œuvres musicales qui font référence à la guerre, de tout temps, certes, mais surtout au $\mathrm{XX}^{\mathrm{e}}$ siècle. Pourquoi la musique plus que la littérature ou la peinture ? A cause de son caractère immatériel, vous ne pouvez rien saisir, vous ne pouvez pas la toucher comme vous pouvez toucher une toile ou un livre, vous ne pouvez que vous laisser envahir, comme dans la guerre, c'est une question de vie ou de mort, comme dans la catastrophe, car il s'agit du corps, vous ne percevez la musique que par le corps [...] (37, je souligne).

Voyons comment cette expérience physique, sensible de la musique est vécue au niveau de la réception, par la narratrice du récit. En particulier, nous essayerons de mieux comprendre son écoute de la $10^{\mathrm{e}}$ Symphonie de Chostakovitch.

\section{L'écoute de la narratrice: une ekphrasis musicale}

C'est étendue sur son lit, les yeux fermés, que la narratrice écoute cette symphonie de Chostakovitch: ainsi, elle s'ouvre physiquement et mentalement à la musique, «glissant dans le mouvement d'ensemble, l'atmosphère» (34). Cette traversée de la symphonie, elle va la raconter par une évocation très précise et détaillée, que nous appellerons une ekphrasis. Dans l'Antiquité, l'ekphrasis était un exercice rhétorique consistant à produire un discours qui permette à l'auditoire de voir mentalement, donc d'imaginer l'objet décrit. Cet objet pouvait être un lieu, une époque, un événement, une personne... Comme le montre Ruth Webb (2009), ce n'est que beaucoup plus tard, à partir de la deuxième moitié du $\mathrm{XX}^{\mathrm{e}}$ siècle, avec le célèbre essai de Leo Spitzer sur le poème de Keats, «Ode on a Grecian Urn», que l'ekphrasis prend le sens restreint de description d'une œuvre d'art visuelle. Elle devient alors «la représentation verbale d'une représentation visuelle, description qui implique, selon les mots de Théophile Gautier, une 'transposition d'art'» (Webb 2009, 34). C'est alors aussi que naît l'ekphrasis musicale: tentative, par la description, donc par les mots, de communiquer l'expérience de l'écoute, de la faire vivre émotionnellement et peut-être même entendre mentalement au lecteur. L'ekphrasis musicale a certes une longue histoire dans la littérature mais le plus souvent—songeons à Verlaine, à Proust, à Thomas Mann-elle décrit des œuvres musicales imaginaires. Dans le roman de Wajsbrot par contre, il s'agit d'œuvres réelles, ce qui rend l'exercice de l'ekphrasis d'autant plus exigeant. Voyons comment cela fonctionne: de quelle manière, par quels procédés littéraires, la narratrice décrit-elle ses impressions? Et plus largement, comment est-il possible de parler de musique?

Dans sa description, la narratrice suit l'ordre des différents mouvements de la symphonie. Le premier mouvement est un Moderato: «L'importance des bois, le dialogue avec les cordes, l'ampleur de la mélodie et la tonalité mineure, tout concourait à l'impression de gravité, tout confirmait ce qu'avait dit le maître». (34) Le maître lui a en effet expliqué que la $10^{\mathrm{e}}$ Symphonie de Chostakovitch a été écrite pendant l'été 1953, peu après la mort de Staline, en mémoire des victimes du stalinisme. Dès lors, tout concorde, dans cette pièce musicale, à parler à la narratrice 
de la catastrophe du stalinisme, «un naufrage aux conséquences incalculables» (36). L'écoute de la narratrice est donc d'emblée orientée par un savoir objectif, historique, sur le contexte de la musique.

Cependant, cette citation révèle qu'il y a un autre élément qui est indispensable à l'ekphrasis musicale, c'est la connaissance de la musique elle-même, de ses techniques: l'harmonie, l'apport des divers instruments, la mélodie, le rythme, la dynamique... La pièce musicale fait naitre une impression, une émotion subjective-la gravité-mais pour être juste, celle-ci doit trouver son soubassement dans des éléments objectifs: le contexte historique, le rôle respectif des bois et des cordes, le mouvement de la mélodie et le fait que la symphonie est en si mineur. Il en est de même lorsque, plus loin, la narratrice décrit un de ces paroxysmes typiques de Chostakovitch:

Tout à coup, le volume augmentait, les cymbales scandaient - on pouvait croire entendre des voix. Puis la mélodie diminuait, augmentait de nouveau comme des vagues successives envahissant les rives, les percussions sonnaient comme un tambour d'exécution puis les cuivres, annonçant quelle victoire, quelle défaite - les dissonances éclataient et le chant incantatoire revenait, derrière les cordes, des voix dissimulées mais inexistantes, seulement créées par la force de l'orchestre. (35, je souligne).

L'écoute musicale exige donc une oreille éduquée, capable de distinguer le rôle des différents instruments dans les variations de volume et de timbre (cymbales, cuivres), de suivre le mouvement de la mélodie et de percevoir l'harmonie des accords ou les dissonantes. Cependant, ce savoir objectif ne fait que corroborer et enrichir les impressions visuelles causées par la musique, qui sont exprimées ici par la comparaison: les mouvements de la mélodie sont «comme des vagues successives», les percussions sonnent «comme un tambour d'exécution» (autre allusion aux victimes du stalinisme). Un peu plus bas, c'est par l'image, par la métaphore que s'expriment ces impressions visuelles:

Peu à peu l'intensité retombait, une paix revenait, une douceur menacée par le roulement discret des timbales sonnant comme un avertissement, une paix revenait qui était peut-être celle de la mort, un paysage de ruines, la dévastation, partout où porte le regard, l'aube qui se lève après la nuit. (35, je souligne).

Il y ici un art consommé de l'ekphrasis: la musique provoque des images visuelles chez l'auditrice, qu'elle traduit en mots en se servant à son tour d'images, de métaphores. La syntaxe vient renforcer la vivacité de la description (l'enargeia propre à l'ekphrasis ${ }^{3}$ ). Cet ordre est celui de la parataxe, qui reproduit l'ordre (ou le désordre) dans lequel les images défilent dans l'esprit: celui d'une simple association. Ces images sont des métaphores de la fin des temps, étroitement liées au contexte

\footnotetext{
${ }^{3}$ Dans la rhétorique, l'enargeia est « l'art de rendre présentes les choses absentes», c'est-à-dire de créer dans l'esprit de l'auditeur une image vivante de ce dont on parle. Sur l'enargeia, voir Webb, 2009, chap. 4 .
} 
historique de la symphonie. Mais avec l'image de l'aube qui se lève, apparait la catharsis de la souffrance: à la fin, la pièce laisse la narratrice-donc l'auditeur et par extension le lecteur-«soulagé, apaisé, épuisé.» (35). C'est peut-être le sens aussi de ces voix, de ce «chant incantatoire» que perçoit la narratrice, impression vocale qui est créée par des moyens purement instrumentaux.

Dans sa description, la narratrice parvient donc à mettre en rapport ce qu'elle sait de l'œuvre (son contexte biographique et historique) avec la forme musicale, et à traduire cette unité par une forme verbale, littéraire, grâce à des procédés poétiques (métaphores, comparaisons, syntaxe...). Cependant, au terme de l'écoute, elle se demande quel est l'apport de cette connaissance du contexte d'une œuvre: rend-il «plus authentiques, [ou au contraire] plus fausses» les impressions causées par la musique (36) ? Ni l'un ni l'autre, semble-t-elle dire, car, de par son immatérialité, la musique n'est pas descriptive: elle ne raconte aucune histoire et ne parle de rien (ce qui ne veut pas dire qu'elle est autoréflexive, détachée du réel). Aussi la succession de sensations et d'impressions visuelles traversées par la narratrice est-elle indépendante de ce contexte historique.

Cette autonomie de l'expérience musicale apparait clairement plus loin dans le roman, lors de l'enterrement du maitre. On y joue une pièce musicale parfaitement inconnue de la narratrice, et qui l'impressionne profondément: elle lui semble la quintessence de la figure du maitre, «le vrai portrait du maitre» (119) Ce n'est qu'après coup qu'elle découvre qu'il s'agit de Coro de Luciano Berio. Sur le moment, l'écoute musicale est donc dénuée de tout savoir, de tout contexte. Cependant, comme pour la symphonie de Chostakovitch, les impressions auditives et visuelles de la narratrice sont basées ici sur des observations, sur des perceptions musicales très précises. Ainsi, elle distingue un rythme «résonnant parfois comme une cloche, le tambour d'une fête rituelle». Dans son imagination, les phrases musicales et la dynamique de la pièce sont visualisées: «Les mouvements ascendants, les crescendos que j'entendais, j' avais l'impression de les voir comme des lignes mouvantes, des vagues qui s'élevaient et s'abattaient tandis que le chœur des hommes tentait de rester, sur ses esquifs fragiles, à la surface de l'océan.» (119).

On retrouve ici, en forme de métaphore filée, la comparaison—assez courantede l'écoute musicale à la navigation en mer, en haute mer ! Cependant, l'ekphrasis de Berio ajoute un nouvel élément, qui est la réflexion sur l'essence de la musique, dans ses rapports avec les autres arts et le langage:

Tout à coup, je comprenais la force, le sens de la musique, sa capacité à exprimer nos chemins les plus profonds, nos détours, en leur donnant une autre dimension qui, échappant aux moyens habituels de l'expression, de la pensée, les transcendaient tout en les accomplissant.» (119, je souligne).

Ces termes-une autre dimension, la transcendance-renvoient à une conception de l'art proche de celle du Romantisme allemand, qui considère l'œuvre d'art comme l'expression, comme la réalisation de l'Absolu. Par l'Absolu, les Romantiques entendaient l'unité, l'harmonie des contraires. Or celle-ci est réalisée, «accomplie», dit la citation, par et dans l'œuvre d'art. Celle-ci ouvre la voie à une dimension supérieure, littéralement absolue c'est-à-dire séparée du réel, où se rejoignent la nature et l'esprit, le sujet et l'objet, la forme et le contenu. Pour les philosophes 
romantiques-Fichte, Schelling — mais aussi les grands poètes de l'époque comme Schiller, Schlegel, Novalis, Hofmannsthal, l'œuvre d'art constitue la totalité vivante de la forme et du contenu. ${ }^{4}$ C'est cela qui fait que l'œuvre d'art a un statut à part, transcendant par rapport à la pensée et au langage. Plus tard, Schopenhauer viendra-et en France Mallarmé—-souligner la place privilégiée de la musique comme l'art le plus profond, le plus métaphysique, et supérieur en cela à la poésie et aux autres arts. Cependant, il ne semble pas qu'il y ait une telle hiérarchie des arts dans le cycle 'Haute mer', qui visite tour à tour tous les arts, et tous les médias contemporains, sans en privilégier aucun—sauf peut-être la littérature.

Dans la phrase de Wajsbrot que je viens de citer, j'entends un écho de cette conception romantique de la musique. Cet écho serait parfaitement en accord avec les allusions au Romantisme allemand ailleurs dans son œuvre, comme par exemple le remarquable portrait du peintre Kaspar David Friedrich, dans Caspar Friedrich Strasse (2002). Avec ses descriptions de pièces musicales dans Conversations avec le maitre, Wajsbrot pose à nouveau, de manière tout à fait contemporaine, le paradoxe de toute ekphrasis musicale: si la musique, comme elle semble le dire ici, est un art qui échappe «aux moyens habituels de l'expression, de la pensée» (119), donc du langage, l'expérience de la musique, sa réception est pourtant uniquement dicible par le langage, par les mots, par la forme littéraire, telle que l'auteur la pratique ici de manière exemplaire. En outre, dans son savant enchevêtrement de l'intériorité et du monde extérieur, le roman met en scène un art-création mais surtout réception—qui est en consonance avec le réel et l'Histoire, avec la souffrance.

Open Access This article is licensed under a Creative Commons Attribution 4.0 International License, which permits use, sharing, adaptation, distribution and reproduction in any medium or format, as long as you give appropriate credit to the original author(s) and the source, provide a link to the Creative Commons licence, and indicate if changes were made. The images or other third party material in this article are included in the article's Creative Commons licence, unless indicated otherwise in a credit line to the material. If material is not included in the article's Creative Commons licence and your intended use is not permitted by statutory regulation or exceeds the permitted use, you will need to obtain permission directly from the copyright holder. To view a copy of this licence, visit http://creativecommons.org/licen ses/by/4.0/.

\section{Références}

Wajsbrot, C. (2004). Beaune-la-Rolande. Paris: Zulma.

Wajsbrot, C. (2007). Conversations avec le maître. Paris: Denoël.

Wajsbrot, C. (2008a). Le son du silence. Romanisches Zeitschrift für Literaturgeschichte, 32, 241-254.

Wajsbrot, C. (2008b). Après coup. In A. Schulte Nordholt (Ed.), Témoignages de l'après-Auschwitz dans la littérature française d'aujourd'hui (pp. 25-29). Amsterdam: Rodopi.

Webb, R. (2009). Ekphrasis imagination and persuasion in ancient rhetorical theory and practice. Routledge: Farnham.

Publisher's Note Springer Nature remains neutral with regard to jurisdictional claims in published maps and institutional affiliations.

\footnotetext{
${ }^{4}$ Cela ramène à la citation de Hofmannsthal faite par Wajsbrot dans « Le son du silence», et citée plus haut.
} 